increase rapidly. It appears, then, that the modificarions in diet and in the working conditions of the prisoners have produced the effect of materially reducing the incidence of beri-beri, and with it the death rate.

\section{Experiments on Fowls.}

In the meantime experiments on fowls were undertaken, with the design of throwing light upon this and other questions. The first experiments included one which would show whether sunlight was capable of preventing the development of polyneuritis in fowls fed on a diet of highly polished rice and water; and also one to show whether exercise was capable of preventing such development.

\section{The Effect of Sunlight.}

Fowls were exposed to direct sunlight for several hours daily, while others were kept constantly in the dark, the conditions being otherwise equal. The fowls in the two categories died in the same time as did control fowls exposed to a normal indoors variation of light and dark. No evidence of any preventive effect of sunlight or any contrary effect of lack of sunlight was obtained.

\section{The Effect of Exerciso.}

Fowls were kept in two batches-the first in individual small cages, in which there was no room for free movement, the second in a large run where free movement was possible. The difference in the periods of survival was striking; all the fowls deprived of exercise died in seventeen days or less, whereas of those which had free movement none succumbed in less thah thirty-one days.

The exercise experiment was considered of importance and was repeated. The conditions were as follows:

Each animal of batch number one was placed in a separate cage of such size that, while the fowl had ample room to turn round and stand upright and feed, it was prevented from obtaining exercise. The cages were placed in a large room, well ventilated and well lighted. The fowls of batch number two were allowed to roam free in the room and were provided with a stick on which to roost some feet above the floor. The room and cages were thoroughly washed before starting the experiment, and each day the floor of the room and the cages were swept out and dusted with clean sand. The small cages permitted slightly more movewent, while the room space permitted much more exercise than in the first experiment. The diet, as before, consisted of highly polished rice and water. The results of the experiments were :

Of five fowls fed on polished rice and water none survived

longer than twenty-six days when deprived of exercise.

of five fowls fed on polished rice and water all survived thirty-eight days or longer when allowed free exercise.

A control experiment was carried out in order to determine whether lack of exercise would adversely affect fowls which were given a normal diet; for this purpose the same small boxes were given a normal diet; for this purpose the same small boxes were
used. Of five fowls kept in these boxes under identical conditions except as regards diet, none died in a period of three months; on the contrary, all kept in excellent condition and increased greatly in weight.

The conclusion to be drawn from these experiments is that, given a group of animals which are subsisting on a diet containing insufficient anti-beri-beri vitamin, lack of exercise forms a very important factor in precipitating the ultimate result.

The results of the two experimental investigations in fowls confirm thus in a most definite and convincing manner the conclusion arrived at as the result of clinical observation in man-namely, that lack of exercise played an important part in precipitating the occurrence of beri-beri in tailors in Freetown Prison.

Attenipts were made to ascertain whether this factor may not have produced effects of a similar kind in other epidemics of beri-beri. An observation on this point is made by Lasnet in .Senegal. Lasnet found that, in the gaol at Dakar, military prisoners who were exempt from outdoor duty developed beri-beri in greater proportion than other classes of prisoners. It is noted that when the disease made its appearance for the first time compulsory exercise was invariably followed by an amelioration of symptoms. Davidson $^{2}$ says that the disease attacks men on active service far less severely than those in barracks.

Lack of exercise is frequently mentioned in a general way as a contributory cause, especially when coupled with overcrowding and insanitary surroundings, but it does not seem to have been recognized hitherto that lack of exercise has such a definite effect as has been brought into evidence by the observations and experiments here recorded. It is probable, however, that this factor plays a part which may in some cases be of great importance. It may, for example, serve to explain such facts as surgical beri-beri occurring in patients lying in hospital. The records of epidemics are as a rule of little service in throwing light upon the effect of this factor, as they do not afford information as to the amount of exercise taken by those affected by the diseasethe significance of this not having been realized. Such records, however, as those given by $\mathrm{Greig}^{3}$ seem to suggest that lack of exercise may have had a definite influence. Under "Occupations of persons attacked by epidemic dropsy" he gives a table for males. This table shows that in 293 cases the highest incidence was in clerks (104). Greig considers that the extensive outbreaks of epidemic dropsy are determined by a sustained high price of food grains, and that "it is just those persons with small fixed incomes who would be most severely affected." In this way he explains the high incidence among the clerical, artisan, student, or petty trader classes. While it is difficult to assign a proportional value to the figures given, the fact that a sedentary occupation, such as that of clerks, yields such high figures of epidemic dropsy is suggestive. Clerks cannot be regarded as the class who are most adversely affected in their diet by an increase of the cost of grain.

Beri-beri is notoriously a disease which occurs in institutions and in places where a fixed diet and some degree of confinement are almost constantly present-for example, prisons, asylums, ships, etc. It is important for those in charge of institutions to realize that such useful occupations as tailoring, mat-weaving, and basket-making are not such as to provide sufficient exercise.

\section{Summary.}

1. Observations of an epidemic in Freetown Prison suggested that lack of exercise played an important part in determining an attack of beri-beri where the necessary predisposing conditions of diet existed.

2. Experiments on fowls showed clearly the decisive influence of lack of exercise in accelerating death in animals fed on a diet deficient in vitamin $B$.

3. In previous beri-beri epidemics this factor has been almost entirely overlooked.

4. The fact that lack of exercise accelerates and accentuates the ill effects of a diet deficient.in vitamin $\mathbf{B}$ opens up an interesting problem in metabolism.

REFERENCES.

${ }^{1}$ Lasnet: Lancet, April 3rd, 1897, p. 973. 2 Davidson: Edin. Med. Journ., August, 1881. ${ }^{3}$ Greig, E. D. W.: Sci. Mem. Off. Med. and San. Dept. Gov. India, No. 49, p. 10.

\section{THE INTRANASAL OPERATION FOR DISEASE OF THE LACRYMAL APPARATUS.}

\author{
$\mathrm{BY}$
}

W. J. HARRISON, M.B., M.R.C.S.,

SURGEON, EAK, NOSE, AND THROAT HOSPITAL; SURGEON IN CHARGE, THIROAT DEPARTMENT, HOSPITAL FOR SICK CHILDREN, NE WCASTLE-UPON-TYNE.

THE treatment of disease of the lacrymal apparatus has always been full of disappointments both to the ophthalmologist and the patient. The rhinologist has ignored it, although a considerable proportion of the cases of disease of this system originate in the nose. In consequence it has been left to the ophthalmic surgeon to do the best he could with probes, styles, and excision of the sac. The introduction of drainage of the sac through the nose by one or other of the operations now performed has modified the treatment considerably and to a very great extent done away with the troublesome effects of excision of the sac. The cases must be properly selected, as the operation is devised for disease arising from stenosis of the lacrymal duct, and is useless for epiphora due to some defect of the puncta or canaliculi, facial paralysis, or disturbed secretory function of the gland.

For mucocele or suppuration of the sac the operation offers a method much preferable to excision of the sac, doing away with the unpleasant epiphora which remains after the latter operation. For some reason the intranasal operation has not made as rapid an advance as it deserves, though a steadily increasing number of cases are being operated on 
in this way. Its advantages, according to West, are as follows :

1. The physiological function of the path for tears is again restored, so that not only a suppuration of the sac a lacrymal fistula, or a phlegmon is healed, but also the tears flow normally through the nose. A later epiphora is accorfingly avoided.

2. A so-called cure by probing is rendered unnecessary.

3. The lacrymal gland is spared.

4. A skin incision or curetting from without with eventual scar formation is avoided.

Looking briefly at the anatomy of the lacrymal apparatus, we find a punctum at the inner angle of the upper and lower eyelids, each opening into a canaliculus; these unite and enter the lacrymal sac at about the middle of its orbital or external wall. The sac terminates below in the nasal duct, which runs downwards and opens by a valve-like opening below the anterior part of the inferior turbinate. The sac lies in the lacrymal groove and is separated from the interior of the nose by the nasal mucous membrane and the posterior part of the ascending process of the superior maxilla anteriorly and the lacrymal bone posteriorly. Occasionally a forward ethmoid cell is found over the sac, the position of which is just in front of the middle turbinate bone. The duct does not always run in a straight line down from the sac, but may bend at an angle, and in such cases probing is more likely to do harm than good.

I have operated on sixteen patients, in four of whom it was necessary to open both sacs, so that the series comprises twenty operations. These have been cases of epiphora, of mucocele of the sac, and of suppuration of the sac with and without fistula and phlegmon. The operation performed has been that advised by West with slight modifications of no importance.

The sac is washed out and a few drops of cocaine injected. The nose is packed twenty to thirty minutes before the operation with ribbon gauze wrung out of 20 per cent. cocaine and 10 per cent. resorcin. The position of the sac is first measured off outside the nose, and then marked on the mucous membrane if there is any difficulty in locating it without doing this. It is only in narrow noses that this difficulty arises. A very full curved incision is then made down to the bone, and the flap reflected backwards and most of it removed. The bone is then removed in thin layers; this is the most difficult part of the operation, as there is a great tendency for the chisel or gouge to slip. If one attempts to remove large pieces of bone at a time there is danger of considerable splintering of the ascending process of the superior maxilla, causing unnecessary trauma and increase of granulation tissue, the consequent narrowing of the opening being the cause of a disappointing result. Sometimes, owing to the shape of the ascending process, a very considerable thickness of the bone has to be removed. In all cases the opening in the bone should be made as large as possible, not only from above downwards but from before backwards. When sufficient of the ascending process of the superior maxilla has been removed the opening can be enlarged by Killian-Grunwald and Watson-Williams punch forceps. A fine probe passed through the lower canaliculus will push the sac wall through the opening like a tent. It can then be caught with a fine hook and a large piece cut out of its wall or caught with punch forceps and an opening punched out. The opening can then be enlarged with punch forceps till the probe will pass horizontally into the nose and freely in all directions. The base of the flap which was resected is then turned over the posterior edge of the opening. This portion of the flap has not been kept in all cases and its retention does not appear to be a matter of importance. The sac is washed out daily for a few days, the nose douched, and granulations destroyed if they tend to become large. If the operation is successful, discharge from the punctum and lacrymation ceases, and in twentyfour hours the inner angle of the eye only shows norinal moisture. If the result is not going to be good, in somo cases there will be a gathering of tears at this point, although the passage is free to the probe and the flow of lotion.

I have only been able to trace the result in fifteen operations. Of these, twelve say that they are completely cured and have no epiphora. One case of double suppuration with fistula has written saying that she still has discharge and epiphora, but has not had any " abscesses," and one has epiphora. The latter case has been seen, and passing a probe shows the opening to have closed. This sometimes happens (it has occurred in two of the twelve cases who are free from symptoms) and the opening has to be remade. One woman, not included in the above number, when seen about three months ago, said that if she went out in the morning she had epiphora, which stopped when she went home and did not recur if she went out again. This seems to be rather strange, but she held to the statement as being correct. Of the other cases nothing is known, but one had a second operation owing to recurrent suppuration four to five months after the first operation. She left hospital apparently cured, but the final result is not known.

In all the cases in which a fistula was present it healed rapidly. I have never operated on a case during an acute attack of inflammation, but have done so when the symptoms were subsiding. The operation is not difficult; in a few cases it is necessary to remove the anterior end of the middle turbinate or to correct a septal deviation before the area of operation can be reached.

Failure may occur, owing to the opening not being sufficiently large in all directions in the first place, or to free granulations springing up which cannot be kept down, and all surgeons have had cases in which the opening had to be remade. After the second operation the granulations do not appear to be so vigorous. According to Frieburg the canaliculi can normally force tears along to the sac, functioning like a regularly working pump. If this power is lost lacrymation will continue, and amongst the reasons given for this loss of function are: $(a)$ long or improper slitting of the canaliculus; (b) long-continued probing or the use of too large probes; $(c)$ cicatricial alterations of the surrounding soft parts causing impairment of the muscular apparatus.

Statistics vary; I have been able to collect the following list showing the percentage of successes of various operators:

$$
\begin{aligned}
& \begin{array}{llllllll}
\text { West } \ldots & \ldots & \ldots & \ldots & \ldots & \ldots & \ldots & 90 \text { per cent. }
\end{array} \\
& \begin{array}{lcccccccc}
\text { Frieburg } & \ldots & \ldots & \ldots & \ldots & \ldots & \ldots & 90 & \text { per } \\
\text { Polyak } & \ldots & \ldots & \ldots & \ldots & \ldots & \ldots & 71 & , \\
\text { Fraser (in } & 50 & \text { cases) } & \ldots & \ldots & \ldots & \ldots & 85 & ,
\end{array} \\
& \text { In simple cases } \ldots \text { anes of phlegmon and those with } \\
& \text { cicatrices and fistula and those with }
\end{aligned}
$$

The operation offers considerable advantages over excision of the sac, but the question whether it is safe subsequently to perform an operation for cataract or one involving the opening of the eye is open to doubt, as some infection of the sac persists after the operation in some cases.

\section{LYMPHATICOSTOMY IN PERITONITIS.}

BY

ARTHUR COOKE, F.R.C.S. SURGEON TO ADDENBROOKE'S HOSPITAL, CAMBRIDGE.

To Costain ${ }^{1}$ we are indebted for the suggestion that opening and draining the thoracic duct may assist surgical measures in serious cases of general peritonitis. He assumed that the chief cause of toxaemia in peritonitis was the flow of toxic lymph into the left subclavian vein, and went far to prove this in dogs suffering from gangrenous appendicitis by diverting this stream to the surface of the neck. He published a successful case of lymphaticostomy in a girl with pneumococcal peritonitis.

In the following case recovery ensued after lymphaticostomy in an attack of peritonitis which was apparently hopeless. Opinions naturally differ as to what is hopeless, but experience has led me to consider that the type in question has a mortality of over 90 per cent. I may ad I that I look on the ordinary severe appendicitis; with considerable pus in the lower abdomen, with reasonable optimism, and leave the case reported hero to speak for itself.

An agricultural labourer, aged 48, was admitted to Adden. brooke's' Hospital on April 15th, 1924 , with' a history that on April 13th heital on April 15th, 1924, with a hominal pain. In spite of that he got up and milked the cows. His bowels acted, but he became progressively worse, with pain and vomiting on April 14th, and was admitted to hospital on the afternoon of 